\title{
Acoustic Flame Suppression Mechanics in a Microgravity Environment
}

\author{
Eryn Beisner ${ }^{1} \cdot$ Nathanial David Wiggins ${ }^{2}$. \\ Kwok-Bun Yue ${ }^{2} \cdot$ Miguel Rosales $^{2} \cdot$ Jeremy Penny $^{2}$. \\ Jarrett Lockridge $^{2} \cdot$ Ryan Page $^{2} \cdot$ Alexander Smith $^{2}$. \\ Leslie Guerrero $^{2}$
}

Received: 17 August 2014 / Accepted: 13 March 2015 / Published online: 28 March 2015

(C) Springer Science+Business Media Dordrecht 2015

\begin{abstract}
The following paper deals with acoustic flame suppression mechanics in a microgravity environment with measurements taken from an Arduino-based sensor system and validation of the technique. A Zippo lighter is ignited in microgravity and then displaced from the base of the flame and suppressed using surface interactions with single tone acoustic waves to extinguished the flame. The analysis of data collected shows that the acoustic flame suppression measurementtechniques are effective to finding qualitative differences in extinguishing in microgravity and normal gravity. Further, the results suggest that the suppression may be more effective in a microgravity environment than in a normal $(1 \mathrm{~g})$ environment and may be a viable method of extinguishing fires during space flight.
\end{abstract}

Keywords Acoustic flame suppression - Arduino sensors $\cdot$ Cold plasma physics $\cdot$ Microgravity study

\section{Introduction}

Previous experiments and numerical simulations of acoustic effects on fire have shown that both frequency and sound velocity may affect the surface of a flame (DARPA 2008; Feynman 2012; Fialkov 1997). Here a single bass

\footnotetext{
N. D. Wiggins

Nathanial.wiggins@sjcd.edu

1 Barrios Technology, Houston, TX 77058, US

2 University of Houston-Clear Lake, Houston, TX, US
}

tone is used to suppress a homogeneous flame in a microgravity environment. An Arduino-based sensor system is used for analysis along with video cameras for visual inspection. The photos show that the flame is displaced greater with lower frequencies while the fire sensor data indicates that the flame is more greatly affected in the microgravity environment by specific tones. Results and discussion are included with recommendations for future studies.

\section{Data Capturing}

The data capturing system used in the experiment is constructed from an Arduino development board and various sensors whose datasheets are all available in the references section. All programming was done using institutional resources and the GoPro Hero cameras are set as reference for aid in the analysis. Numerical simulations indicate a horizontal velocity induced in the flame and displacement of the flame tip as a result of the acoustic wave (Plaks et al. 2005), therefore both primary cameras are set in positions perpendicular to the direction of the propagation for analysis and for validation of visual data.

The accelerometer used is an MPU 6050 manufactured by Invensense and implemented on a GY-87 Arduino sensor that possesses 3 degrees of freedom for detecting acceleration. Open source libraries are used to log data from the MPU6050 using IIC communication protocol via pins A5 and $\mathrm{A} 4$ on the Arduino board. This enables direct reading of acceleration values which are immediately converted to G-forces. A Kalman filter can be applied to normalize the acceleration data and reduce noise from internal and external sources, but raw data is sufficient for parabolic analysis (see Figs. 1 and 2). 
Top View

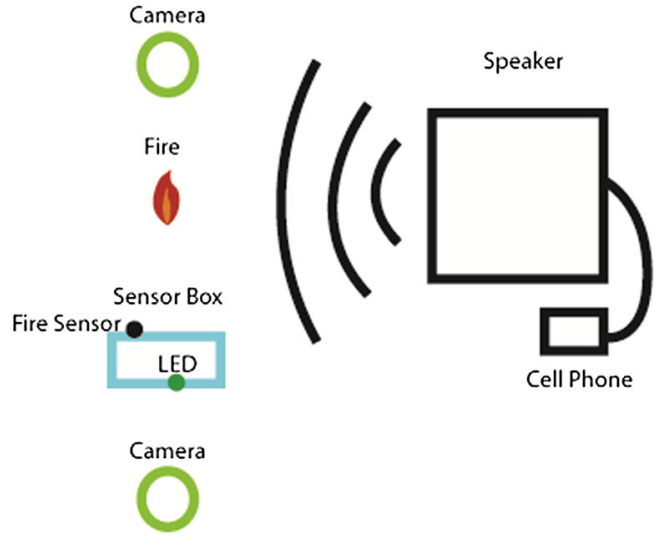

Fig. 1 The figure above shows a top view diagram of the experiment setup. It consists of a sub-woofer, a cell phone with a tone generator application, a modified zippo lighter to hold the flame, an Arduino board with flame sensor, accelerometer, and a bi-color LED
The accelerometer output is converted to multiples of gravitational pull and graphed in comparison to the iteration value in thousands where 100 iterations equal 5 seconds. Although noise is apparent in the system, the parabolas are clearly defined structures that are identified in the output algorithm and recorded on the SD card. The output LED provides visual identification for verification in the video. In each parabola, a frequency is tested or retested during the microgravity phase, which generally lasts 20 seconds. If the fire is not extinguished by the end of the 20 seconds of microgravity, it is manually suppressed and extinguished.

The fire sensor used for this experiment is a SENS-FL001 Flame Sensor Module from Future electronics. The Arduino sensor is capable of detecting light from a range of $760 \mathrm{~nm}$ to $1100 \mathrm{~nm}$ with an adjustable detection distance between $20 \mathrm{~cm}$ and $100 \mathrm{~cm}$ and a detection angle of about 60 degrees. The sensor is sensitive to the fire light wave

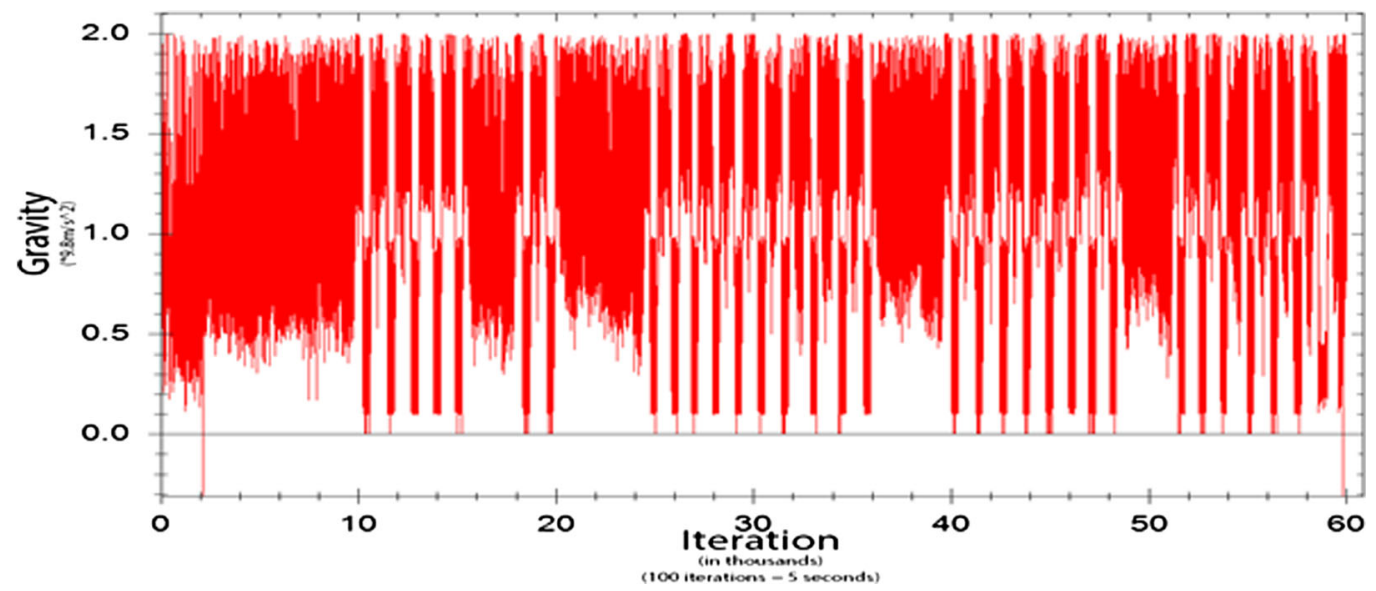

Fig. 2 While the noise from the aircraft makes the exact reading difficult, the maximum hypergravity and the minimum microgravity can be deciphered and the time verified by camera
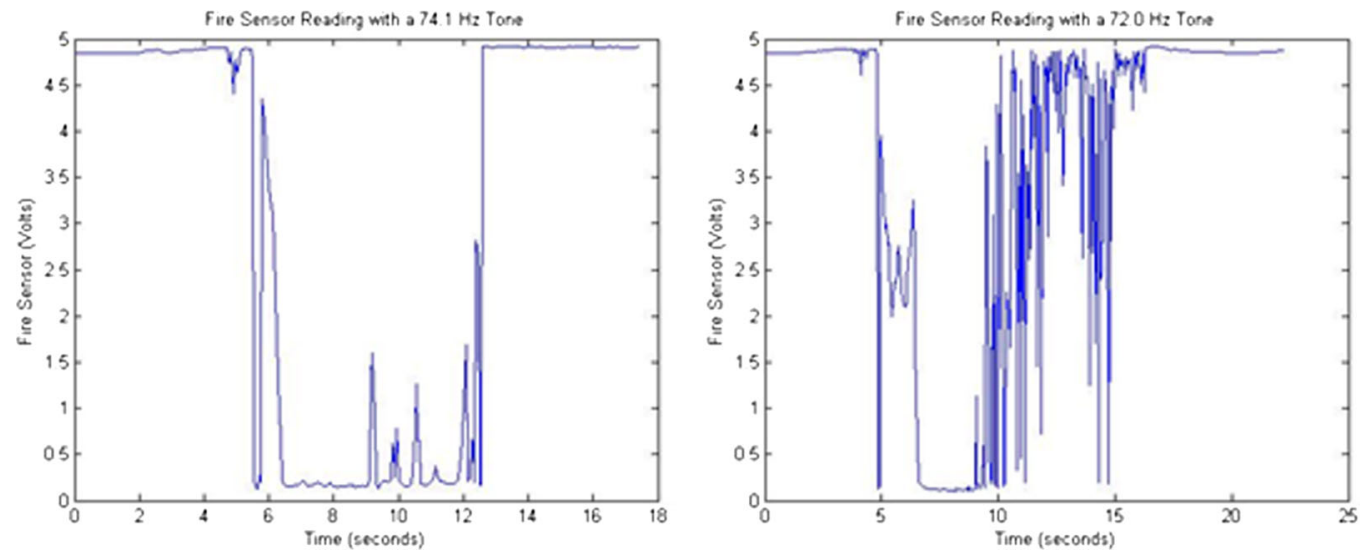

Fig. 3 The analog fire sensor readings in volts are compared to time in seconds. Note that the left panel shows a flame being quickly and effectively while the right panel shows a flame being interacted with for longer periods before being extinguished 


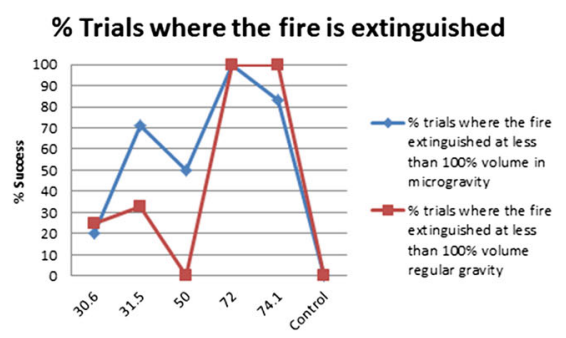

Fig. 4 The frequency versus percentage of trials extinguished shows that the microgravity flame was generally extinguished more successfully than the lab setting. The frequency versus voltage shows the average temperature reading while identifying a flame in microgravity. Note that the higher voltage indicates a
Average Fire Sensor Reading

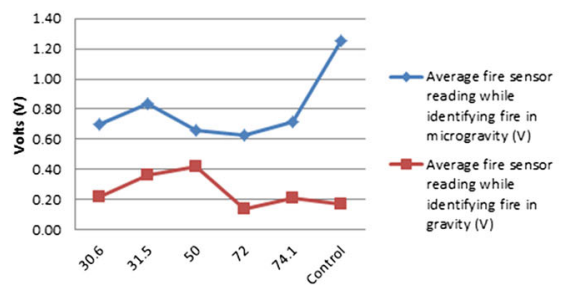

Minimum Fire Sensor Reading

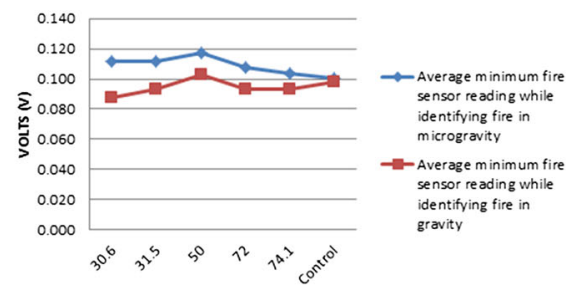

lower temperature. The minimum fire sensor reading versus frequency shows the hottest temperature that a flame could reach while in the presence of acoustic waves. Note that the control frequency has about the same highest temperature in both settings

output of the sensor is a value between 0 and 1023 which can be converted into voltage in order to describe the intensity of the flame. The conversion of the analog values to volts is

performed using a rate of 5 volts/1023 bits. For reference,

spectrum and will output a digital signal when fire is detected by the module. The fire sensor module features two outputs, digital and analog. The digital output will be either a 1 for flame detection or a 0 for no detection. The analog
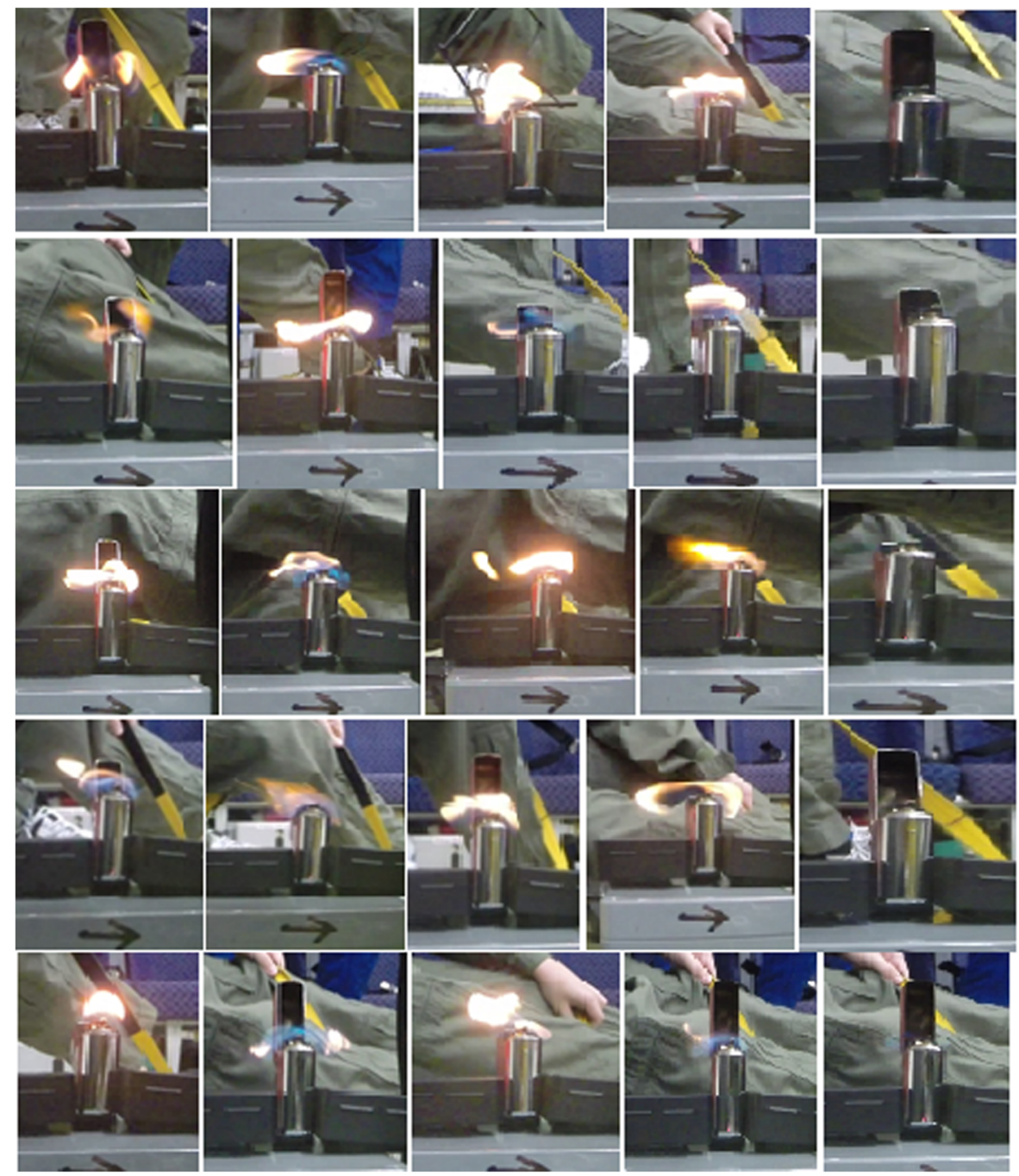

Frequency 30.6

Frequency 31.5

Frequency 50

Frequency 72

Frequency 74.1

Fig. 5 The effects of the experiment on a flame during different intervals of frequency 
a fire with voltage of 0 is a very bright and hot fire while a voltage of 5 volts indicates no fire or intense light is detected, as shown in Fig. 3

\section{Analysis}

Extinction of the flame appeared more effective in microgravity than in normal gravity as shown by the flame being extinguished in less time and at a lower temperature than in normal gravity. With the exception of equipment failure during a trial at $74 \mathrm{~Hz}$ there are no anomalies in the data, and only raw data is shown. Previous experiments involving acoustic flame suppression suggest that there exists optimal surface interactions at two frequencies with methane gas, one low frequency at roughly $30 \mathrm{~Hz}$ and another higher frequency at about $90 \mathrm{~Hz}$ (DARPA 2008; Plaks et al. 2005) (Figs. 4 and 5) .

\section{Conclusion}

According to the data collected, acoustic flame suppression works in both regular gravity and microgravity and the Arduino-based sensor system is effective in gathering data for analysis. The raw accelerometer readings are adequate for reproducing the parabolic motions of the aircraft and the LED is adequate for aligning the data file with timestamps to the video. The fire sensor is able to read the fire with correlated digital and analog outputs, providing a $92 \%$ accuracy in time comparison to the video when half-voltage is used as a baseline. In the microgravity environment, the flame suppression is qualitatively distinct and appears more effective, though further studies are recommended. The flame was suppressed with more efficiency at 74.0 $\mathrm{Hz}$ and was extinguished more quickly at the $30.6 \mathrm{~Hz}$ frequency, although a range of frequencies were effective with adequate acoustic pressure.

\section{References}

DARPA: Instant Flame Suppression. Harvard University (2008)

Feynman, R.: Behavior of Cold Plasma Physics (2012). Available: http://theory.physics.helsinki.fi/ xfiles/plasma/12/lect12/ Cold_plasma_waves_12.pdf

Fialkov, A.B.: Investigations on Ions in Flames. Prog. Energy Combust. Sci 23, 399-528 (1997)

Friedman, R.: Urban Progress in Fire Detection and Suppression Technology for Future Space Missions, Space 2000 Conference and Exposition (2000)

Plaks, D., Nelson, E., Hyatt, N., Espinosa, J., Coley, Z., Tran, C., de Mayo, B.: Zero-g Acoustic Fire Suppression System. J. Acoust. Soc. Am. 118 (2005)

Tunabe, M., Yano, T., Kuwahara, T.: Numerical Simulation on the Flame Propagation in Acoustic Fields. JASMA 23, 371-375 (2008)

BMP180: http://www.adafruit.com/datasheets/BST-BMP180-DS00009.pdf

HMC5883L: http://www.adafruit.com/datasheets/HMC5883L 3-Axis_Digital_Compass_IC.pdf

MPU6050: http://www.invensense.com/mems/gyro/documents/ PS-MPU-6000A-00v3.4.pdf

Fire Sensor: http://www.fut-electronics.com/wp-content/plugins/fe downloads/Uploads/Flame-sensor-arduino.pdf

Bicolor LED: http://www.lc-led.com/products/n500tgr4d.html 